Ger J Exerc Sport Res 2020 · 50:480-486 https://doi.org/10.1007/s12662-020-00667-6 Eingegangen: 26. Mai 2020

Angenommen: 9. Juli 2020

Online publiziert: 10 . August 2020

๑) Der/die Autor(en) 2020

Titel und Gegenstand dieser Arbeit erhalten ihre Legitimation durch den Beginn der universitär institutionalisierten Sportwissenschaft in Deutschland im Jahre 1920. Es gibt mehrere Argumente, diesen Beginn in Deutschland im Jahr 1920 zu verorten. Da ist zunächst die Gründung der Deutschen Hochschule für Leibesübungen, die am 15. Mai 1920 erfolgte (Court, 2014). Sie geht auf die beharrliche Netzwerkarbeit eines "Männerbündnisses“" (Brinkschulte, zit. n. ebd., S. 40) um Carl Diem und Theodor Lewald zurück. Getragen vom Deutschen Reichsausschuss für Leibesübungen (DRA), dem Dachverband bürgerlicher Turn- und Sportverbände, verfolgte diese "freie Hochschule für Leibesübungen" nach einer Denkschrift Diems zwei Ziele:

1. die „Heranbildung wissenschaftlich geschulter Lehrkräfte für Leibesübungen",

2. die „wissenschaftliche Erforschung aller auf die Theorie, Praxis und Geschichte der Leibesübungen bezogene(r) Gesetze“(Diem, 1919, zit. n. Court, 2014, S. 43).

Die Stärke dieser Hochschule lag in ihrem Netzwerk an Lehrbeauftragten, zu denen im Bereich der Pädagogik auch Eduard Spranger gehörte. In ihrer „Abteilung III“ war die Pädagogik der Leibesübungen angesiedelt, sie stand jedoch im Schatten der experimentellen Psychologie. Ihr erster Leiter Robert Werner Schulte wird heute ,als der erste empirisch forschende Sportpsychologe der Welt“ (Janssen, 2006, S. 140) angesehen. Tatsächlich diente die Forschung im „Psychotechnischen Laboratorium“

\title{
Norbert Gissel
}

Fakultät Für Sportwissenschaft, Ruhr Universität Bochum, Bochum, Deutschland

\section{Jahre Sportwissenschaft in Deutschland - und wo steht die Sportpädagogik?}

der DHfL vor allem der „biopsychologischen“ (Bernett, 1965, S. 56) Typologisierung von Menschen und damit der Selektion. Sie fügte sich so in die vor allem medizinisch-biologisch geprägte wissenschaftliche Gesamtausrichtung der Hochschule ein, die eine ,ingenieursmäßige" Vermessung des menschlichen Körpers zum Ziel hatte (Dickal, 2009, S. 191) $)^{1}$. Pädagogik wurde instrumentell als Mittel zur „Ertüchtigung“ des Volkes angesehen; Leibesübungen seien eine „Lebensfrage für unser Volk“, so der Rektor August Bier ${ }^{2}$. Die DHfL erlangte erst 1931 die staatlicher Anerkennung; Promotions- oder gar Habilitationsrecht besaß sie nicht (Court, 2011, 2014, S. 47) ${ }^{3}$. Sie konnte sich im föderalen System der Weimarer Zeit nicht gegen die Turnlehrerbildungsanstalten durchsetzen, in denen die einzelnen Länder ihren Bedarf an Leibeserziehern ausbildeten. Konkurrenz hatte sie quasi vor der Haustür in Berlin durch die Preußische Hochschule für Leibesübungen, die diese Aufgabe für Preußen übernahm. Konkurrierende Einrichtungen waren weiter die Heeressportschule in Wünsdorf (Eisenberg, 1999, S. 361) sowie die Bundesschule des Arbeiter-Turn- und Sportbundes in Leipzig.

Beeinflusst durch die apologetische Geschichtsschreibung Diems und seiner Schüler wurde der DHfL bislang ein herausragender Stellenwert für die Ge-

\footnotetext{
1 Siehe auch ausführlich F. Becker, 2019, S.317-341.

2 Aus einer Rede des Rektors Prof. Dr. August Bier zur Gründung der Hochschule (zit. n. Court, 2014, S. 137).

3 Einschlägig hierzu:Court, 2011, S.91-99.
}

schichte der deutschen Sportwissenschaft $^{4} \quad$ zugewiesen. Tatsächlich muss aber einem anderen Ereignis eine ebenso epochale Bedeutung zugesprochen werden. Im Liebighörsaal der Universität Gießen wurde am 22. Oktober 1920 ein Institut für Körperkultur gegründet. Damit drangen die Leibesübungen erstmals in den Kanon der wissenschaftlichen Fächer einer Universität ein. Das Institut wurde mit dem Anspruch gegründet, als „Musteruniversität " $\mathrm{zu}$ fungieren, und tatsächlich wurden viele gefundene Lösungen beispielgebend für die Gründung von weiteren Instituten für Leibesübungen, die ab 1925 erfolgten. Aus dieser Gründungsphase liegen verschiedene Denkschriften und Eingaben vor, die sich theoretisch und konzeptionell mit dem Wissenschaftscharakter des Faches, seinen Problemen und einer möglichen institutionellen Verankerung befassten. Besonders sticht eine Denkschrift ${ }^{5}$ des akademischen Turn- und Sportlehrers Dr. Walter Werner ${ }^{6}$ aus dem Jahr 1921 hervor, in der versucht wird, eine pädagogisch ausgerichtete Wissenschaft der

\footnotetext{
4 Eisenberg (1999, S.361, Fn 89) hingegen zitiert den Leiter der preußischen Hochschule Neuendorff. Dieser schrieb, dass bei den Forschungen derDHfL nichtsherausgekommen sei, „was neue Richtungen wies oder neue Bahnen eröffnete". Hier ist allerdings zu bedenken, dass Neuendorff persönlich und institutionell in einer direkten Konkurrenzzu Carl Diem stand.

5 "Ein Institut für Körperkultur an der Universität Gießen. Ein Beitrag zur Ausgestaltung des Instituts". Universitätsarchiv Gießen; Akten der Präsidialabteilung 922-925 (alt 125).

${ }^{6}$ Zur Biographie Werners vgl. Gissel (2006, S. 105 f.).
} 
Leibesübungen zu begründen. Im Kern stand die These, dass Leibesübungen als kulturelles Fach zu begreifen seien. Die damals entwickelte Position soll hier kontrastiert werden mit aktuellen Arbeiten zum Gegenstand, zur Identität und zum Wissenschaftscharakter der Sportpädagogik.

Der Beitrag fügt sich damit in eine Reihe von Versuchen einer disziplinären „Selbstbespiegelung“ ein (Thiele, 2013, S. 29). Zwar wurde verschiedentlich gefordert, die Sportpädagogik solle lieber "handfeste Anwendungsarbeit" (ebd.) hervorbringen, doch zum Anlass eines solchen Jubiläums mag es legitimiert und hilfreich sein, dieser Disziplin, die sich auf dem scheinbar unaufhaltsamen Weg zu einer „normalen Wissenschaft“ (ebd., S. $33 \mathrm{ff}$.) befindet, einen Spiegel vorzuhalten; denn als „normale“ Wissenschaft ist sie auch eine "vergessliche“ (ebd., S. 37) Wissenschaft, ohne ausgeprägte Erinnerungskultur. Getrieben „Von den externen Ansprüchen“ ökonomischer Anreizstrukturen, läuft die Sportpädagogik aktuell Gefahr, sich ihrer „tradierten Problemkonstellationen“ und damit ihrem identifikatorischen Kern zu entledigen (ebd. S. 43).

\section{Aktuelle Analysen und Konzeptionen zum Wissenschaftscharakter der Sportpädagogik}

Die Disziplin Sportpädagogik befindet sich in einem "prekären“ Zustand, so Prohl (2013, S. 11). Sie sei einem „Konkurrenzdruck" durch andere Fächer ausgesetzt, dem sie kaum noch standhalte. Schon vor über 25 Jahren befand Kurz (1992, S. 145), dass es mit der Sportpädagogik „kontinuierlich bergab“ ginge, „wie sich an der Entwicklung von Stellen, Mitteln und Reputation ablesen“ "ließe. Auf sportpädagogischen Professuren säßen ,immer mehr Leute, die das pädagogische Interesse vergessen oder nie gepflegt haben“" (Kurz, 1992, S. 150). Heute findet sich an vielen sportwissenschaftlichen Instituten überhaupt keine professorale Stelle für Sportpädagogik mehr, selbst an solchen, die vorwiegend Lehrer*innen ausbilden; man ist der Meinung, dass eine (empirisch ausgerichte- te) Fachdidaktik reiche. Der Sportpädagogik droht ein ähnliches Schicksal wie einst der Sportgeschichte, die von einer zentralen, identitätsstiftenden Disziplin der Sportwissenschaft in die völlige Bedeutungslosigkeit abgerutscht ist (Luh, 2018). Dem „Verlust pädagogischen Denkens“ (Beckers, 1987, S. 241) in der Sportwissenschaft konnte die Sportpädagogik keinen Einhalt gebieten; geht es um Vermittlungsfragen, werden heute eher Trainingswissenschaftler*innen um Rat gefragt. Zur Praxisrelevanz der Sportpädagogik schrieb Kurz (1992, S. 151): „Wer nichts Brauchbares anbietet, kann nicht erwarten, gebraucht zu werden.“

Der „Umbau der Wissenschaft“ ist also in vollem Gange, „die Geschwindigkeit bemerkenswert" (Thiele, 2013, S. 37). Im Wettbewerb um „Zitationsindices, Publikationsraten, Drittmittelquoten “ droht Fächern, „die den extern vorgegebenen Rahmungen nicht folgen können oder wollen“ (ebd. S. 40) die „Inquisition“" (ebd., S. 36).

Vor drei Dekaden hingegen sah Kurz (1992) in der Sportpädagogik noch die zentrale und übergeordnete Disziplin innerhalb der Sportwissenschaft. Er stritt mit Scherler (1992) und Cachay und Bähr (1992) über die Frage, ob Sportpädagogik als übergeordnete, „undisziplinierte“ Metawissenschaft im Zentrum der Sportwissenschaft stehe und die „Beiträge“ der anderen Disziplinen nach pädagogischen Kriterien $\mathrm{zu}$ prüfen und $\mathrm{zu}$ integrieren habe (Kurz, 1992, S. 151). Kurz argumentierte, dass die ganze Sportwissenschaft unter einer ethischen und pädagogischen Verpflichtung ${ }^{7}$ stehe, dass aber die Sportpädagogik eine „herausgehobene Verantwortung für den pädagogischen Ertrag der gesamten Sportwissenschaft“ (Kurz, 1992, S. 151) habe; sie solle die anderen Disziplinen quasi überwachen. Scherler hielt dagegen, dass sich das Fach damit übernehme: Dies käme einer „Selbstüberhöhung gleich, die sich weder aus ihrer wissenschaftlichen Stellung, noch aus ihrer wissenschaftlichen Leistung rechtfertigen“ lasse (Scherler, 1992, S. 163). Er plädierte dafür, Sportpädagogik als

\footnotetext{
7 Wobei noch zu diskutieren wäre, in welchem Verhältnis Ethik und Pädagogik zueinanderstehen.
}

eine Teildisziplin der Sportwissenschaften (Plural!) anzusehen, die wie jede andere auch einen eigenen identifizierbaren Gegenstand erforschen und mit spezifischen anerkannten Methoden arbeiten müsse. Sportpädagogik könne keine "Nicht- und Metadisziplin zugleich sein“ (Scherler, 1992, S. 171).

Prohl(2013) argumentiert ähnlich wie Scherler für eine disziplinierte Sportpädagogik. Sie müsse ihre „Identität“ finden und diese sei „in Abgrenzung und im Verhältnis zu den übrigen sportwissenschaftlichen Teildisziplinen“ $\mathrm{zu}$ bestimmen, so Prohl (2013, S. 6), ansonsten sei „sportpädagogische Forschung (...) von sportpsychologischer oder sportsoziologischer Forschung nicht zu unterscheiden“ (ebd., S. 11). Prohl ist sicherlich zuzustimmen, wenn er in der Frage nach dem „Sinn“, nach dem „Wozu“ sportlichen Handelns, den Kern sportpädagogischer Reflexionen sieht. Genau an diesem Punkt unterscheidet sich Pädagogik „grundsätzlich von allen anderen (sozial)wissenschaftlichen Disziplinen“ (ebd., S. 11). Hermeneutische ${ }^{8}$ Analysen, Reflexionen und Kritiken „von Menschenbildern $^{9}$ seien eine identitätsstiftende Aufgabe der Sportpädagogik“ (ebd., S. 14). „Es geht vorrangig darum, nach dem Sinn und der Begründbarkeit des Erziehungs- und Bewegungshandelns zu fragen“" (Prohl, 2013, S. 14).

Doch genau an dieser Stelle wird die Sportpädagogik als Wissenschaft (zumindest aus positivistischer Sicht) auch angreifbar. Aus welchen evidenzbasierten Erkenntnissen und nach welchen standardisierten Kriterien kann sie die Frage nach dem Sinn und dem „Wo-

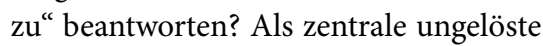
Probleme des Faches identifiziert Prohl so das Normenproblem, die Werturteilsfrage, die Dichotomie zwischen

\footnotetext{
8 "Der Sinn und wünschenswerte Verhaltensweisen sportlichen Handelns können nicht gemessen werden, lassen sich nicht in ein quantifizierbares, den Regeln der Statistik gehorchendes Zahlengebäude eintragen" (Meinberg, 1987, S.33).

9 Allerdings hat nun Giese (2016) darauf aufmerksam gemacht, dass manche in der Sportpädagogikgernezitierten Anthropologien implizit exkludierende und behindertenfeindliche Auffassungen transportieren.
} 
Ger J Exerc Sport Res 2020 · 50:480-486 https://doi.org/10.1007/s12662-020-00667-6

(c) Der/die Autor(en) 2020

\section{N. Gissel}

\section{Jahre Sportwissenschaft in Deutschland - und wo steht die Sportpädagogik?}

\section{Zusammenfassung}

Titel und Gegenstand dieser Arbeit erhalten ihre Legitimation durch den Beginn der universitär institutionalisierten Sportwissenschaft in Deutschland im Jahre 1920. Es gibt mehrere Argumente, diesen Beginn in Deutschland im Jahr 1920 zu verorten. Da ist zunächst die Gründung der privaten Deutschen Hochschule für Leibesübungen, die am 15. Mai 1920 erfolgte. Sie wurde vom Deutschen Reichsausschuss für Leibesübungen (DRA), dem Dachverband bürgerlicher Turn- und Sportverbände, getragen und besaß bis 1931 keine staatliche Anerkennung. Einem anderen Ereignis muss wissenschaftshistorisch vielleicht eine noch höhere Bedeutung zugesprochen werden. Im Liebighörsaal der
Universität Gießen wurde am 22. Oktober 1920 ein Institut für Körperkultur gegründet. Damit drangen die Leibesübungen erstmals in den Kanon der wissenschaftlichen Fächer einer Universität ein. Das Institut wurde mit dem Anspruch gegründet, als "Musteruniversität" zu fungieren. Aus dieser Gründungsphase liegen verschiedene Denkschriften und Eingaben vor, die sich theoretisch und konzeptionell mit dem Wissenschaftscharakter des Faches, seinen Problemen und einer möglichen institutionellen Verankerung befassten. Besonders sticht eine Denkschrift des akademischen Turn- und Sportlehrers Dr. Walter Werner aus dem Jahr 1921 hervor, in der versucht wird, eine kulturalistisch ausgerichtete pädagogische Wissenschaft der Leibesübungen zu begründen. In diesem Beitrag wird die damals entwickelte Position mit aktuellen Arbeiten zum Gegenstand, zur Identität und zum Wissenschaftscharakter der Sportpädagogik kontrastiert. Sowohl Thiele als auch Prohl attestieren dem Fach heute eine prekäre Situation: Zentrale wissenschaftstheoretische Fragen seien ungelöst. War man vor 100 Jahren konzeptionell vielleicht schon weiter?

\section{Schlüsselwörter}

Sportwissenschaft · Jubiläum · Sportpädagogik Interdisziplinarität $\cdot$ Wissenschaftstheorie Kultur

\section{0 years of sports science in Germany —and where does sports education stand?}

\section{Abstract}

The title and subject of this paper are legitimized by the beginning of university sports science in Germany in 1920. There are several arguments for locating this beginning in Germany in 1920. First, there is the founding of the German university for physical education on 15 May 1920. It was supported by the German Reich Committee for Physical Exercise (Deutschen Reichsausschuss für Leibesübungen), which was the association of gymnastics and sports for citizens, and was not recognized until 1931 by the government. Of perhaps even more importance was that on 22 October 1920 an institute for physical culture (Institute für Körperkultur) was founded in the Liebig Auditorium of the University of Giessen. This was the first time that sports education was included in the curriculum of university academic subjects. The institute was founded with the goal to act as a 'model university'. From this founding phase, various memoirs and articles that dealt theoretically and conceptually with the scientific character of the subject, its problems, and possible institutional anchoring. In particular, a 1921 paper from the academic gymnastics and sports teacher Dr. Walter Werner stands out, in which an attempt is made to argue for a culturally oriented sports education of physical exercise.
In the present contribution, the position developed at that time is contrasted with the current work on the subject, identity, and scientific character of physical education. Both Thiele and Prohl state that the subject is in a precarious situation today: central questions of scientific theory remain unsolved. Were we perhaps already further along conceptually 100 years ago?

\section{Keywords}

Sports science · Anniversary · Interdisciplinary studies . Physical education and training . Philosophy of science - Culture formaler und materialer Bildung und schließlich die Frage nach angemessenen Forschungsmethoden.

Angesichts dieser ungelösten Probleme stellt sich die Frage: Wie dachte man vor 100 Jahren über den Wissenschaftscharakter des Faches?

\section{Pädagogik der Körperkultur als Wissenschaft? Überlegungen aus dem Jahr 1921}

Wie konnte so etwas Profanes wie die spielerisch-sportliche Bewegung zum Gegenstand einer Wissenschaft wer- den? Es war, wie Moraw (1991, S. 56) schreibt, sicherlich auch ein Zeichen der Verunsicherung des deutschen Universitätssystems nach dem verlorenen Ersten Weltkrieg und den anschließenden Unruhen, dass ein neues Fach gegen viele Vorbehalte nun in den Kanon der erforschungswürdigen Gebiete aufgenommen wurde. „Denn aus Spiel wird jetzt Ernst!“ (Werner, 1921). Doch wie sollte die spielerische Körperkultur ernsthaft erforscht werden?

Hier haben wir es nicht mit einer Disziplin zu tun, sondern fast alle Disziplinen tragen zur Arbeit bei. Es ist ganz ausgeschlossen, dass der Studierende in selbst 4-6 Semestern hierin einigermassen gründlich arbeiten kann. Er bekommt Stückwerk über Stückwerk. Ein solcher Dr. rer. gymn. - wie er angestrebt wird - wäre das Muster einer Bildung, wie sie nicht sein soll. Rein äußerlich aus allerlei Wissenschaftsgebieten einiges zusammengetragen, dazu technische Kenntnisse in Sport und Turnen - das wäre das Ergebnis des Studiums. (...) Eine Universität darf sich nicht dazu hergeben, solchen geschilderten Dilettantismus zu fördern (ebd.). 
Mit erstaunlicher Schärfe sprach Werner hier das bis heute ungelöste Problem der Multidisziplinarität der Sportwissenschaften (Plural) an. Ein Sammelsurium an sportwissenschaftlichen Einzeldisziplinen, die sich inhaltlich und methodisch v.a. ihren Mutterwissenschaften verpflichtet fühlen, liefern „Stückwerk über Stückwerk“. Sie haben aus der Sicht Werners an einem Institut für Körperkultur nichts zu suchen. „Geschichtliche Forschung, die die Leibesübungen betreffen, gehören der Geschichtswissenschaft nach wie früher an, physiologische Untersuchungen, die wichtig sind für Sport und Turnen, bleiben Arbeiten der medizinischen Fakultät“ (ebd.). Es sei vielleicht pragmatisch $\mathrm{zu}$ rechtfertigen, solche Forschungen an einem Institut für Körperkultur vorzunehmen, im Sinne der „Logik“ der Wissenschaften sei es jedoch völlig unangemessen. Absolventen eines Instituts für Körperkultur müssten mehr sein, „als Leute, die in Physiologie, Verwaltungslehre, Geschichte, Technik der Leibesübungen erfahren sind“. Denn solche seien nur „Trainer" und eine „Verspottung der Wissenschaft“ (ebd).

Gegen die auch heute weit verbreiteten positivistischen Auffassungen der Sportwissenschaften ${ }^{10}$ wandte er ein: „Und gewiss, wenn es sich bei der Arbeit solcher Institute um weiter nichts handelte, als um die beste Ausführung, richtige Dosierung und beste Verwaltung der Leibesübungen, das Misstrauen unserer Geistigkeit wäre in jeder Beziehung gerechtfertigt" (ebd.).

Dem „Dilettantismus“ der multidisziplinären Sportwissenschaften hielt Werner eine kulturtheoretische Position entgegen. Der Brennpunkt der neuen Wissenschaft müsse im Kulturgedanken liegen, definiert als die „Vernunft des Körperlichen“.

Diese hat ihren Sinn in sich selbst. Sie wurzelt in den Tiefen der Menschlichkeit. Nur wer aus dieser Quelle schöpft, kann

10 Thiele $(2013$, S. 37) verweist darauf, dass die Sportpädagogik auf ihrem Weg zu einer "normalen“ Wissenschaft zunehmend zu einer "vermessenen“ Disziplin würde. „Wissenschaftliche Produktion" folge der Logik der „industriellen Warenproduktion“, ihr Erfolg ermisst sich an "numerischer Abbildbarkeit". von Körperkultur reden. Diese Quellen im Menschen zu erschließen, sie zum Bewusstsein zu bringen, ist das letzte Ziel eines Instituts für Körperkultur, dem alles andere untergeordnet ist (ebd., S. 12).

Übersetzt man diese Aussagen in eine moderne Sprache, so ist für die Frage nach der Identität des Faches zunächst auf die anthropologische Grundlegung $\mathrm{zu}$ verweisen, ohne die eine fundierte Bildungstheorie nicht denkbar ist (Meinberg, 1987; Gissel, 2019). Die Objektivationen der Körperkultur wurden „vom Menschen aus seiner Existenz zu seiner Existenz entworfen und geschaffen", so Bernett (1965, S. 11). Mit ihren „Erlebnisweisen, Motiven und Haltungen“" erfüllen die Bewegungsformen grundlegende identifizierbare anthropologische Bedürfnisse.

Eine wesentliche Pointe der Position Werners besteht darin, dass er der kultivierten Körperlichkeit einen Eigensinn zuspricht: Die „Vernunft des Körperlichen“ habe „ihren Sinn in sich selbst“. Damit nimmt er eine Position ein, die diametral konträr zu aktuellen fachdidaktischen Konzepten steht, die propagieren, sportliche Bewegungen müssten durch die Akzentuierung von sog. „pädagogischen Perspektiven“" mit Sinn belegt werden ${ }^{11}$. Viele Sportpädagog*innen verwechseln heute, wie Güldenpfennig (1998) deutlich gemacht hat, die zu respektierenden individuellen Sinnzuschreibungen von (sportlichen) Handlungen auf der subjektiven Seite mit der zu leistenden wissenschaftlichen (Re-)Konstruktion der kulturellen Sinnstruktur des Sports auf der objektiven Seite. Aus kulturtheoretischer und ästhetischer Sicht ist es eben ein Unterschied, ob man - wie etwa üblicherweise in der Sportmedizin - wertneutral von körperlicher Aktivität spricht oder ob man kulturelle Objektivationen in Form von Sportarten $^{12}$ meint, die jeweils einen

\footnotetext{
11 So etwa Kurz (2011, S. 17): „Tatsächlich ist der Kerngedanke, dass Sport nicht seinen Sinn hat, sondern auf unterschiedliche Weise mit Sinn belegt werden kann, alt und eigentlich unbestreitbar."

12 Man beachte den Wortstamm: ars/ artes $=$ Kunst.
}

spezifischen phänomenalen Gehalt in sich tragen. „Jede Sportart hat etwas, was erfreut und befriedigt, sonst wäre sie nicht entstanden" (Volkamer, 2003, S. 12).

Zugleich positioniert sich Werner damit gegen alle Versuche, Leibeserziehung $\mathrm{zu}$ instrumentalisieren. Damals galt es, die Körperkultur gegen medizinisch-rassenhygienische Ideologien $\mathrm{zu}$ verteidigen, die intendierten, einen selektierten, durchtrainierten und militärtauglichen deutschen Volkskörper zu züchten. „Aber so gewiss Wissenschaft nicht Dienerin der Technik ist, (...) so gewiss steht auch für uns die Vernunft des Körperlichen, die Körperkultur nicht im Dienste der Leibesübungen oder von Werten wie Gesundheit und Wehrhaftigkeit."

Im Grunde nahm Werner hier bereits eine zentrale Conclusio der Instrumentalisierungsdebatte (der 1980er und 90er Jahre) in der deutschen Sportpädagogik vorweg: Güldenpfennig (1998, S. 4) machte in einem abschließenden Kommentar deutlich, dass die Stellung des Sports im Fächerkanon der Schule geschwächt würde, „wenn seine spezifische und unersetzliche - ästhetische! Sinnstruktur verkannt, verfehlt oder gar mit Rücksicht auf außersportliche Ziele, die man für wichtiger erklärt, aufgegeben wird“. Gleichwohl sind aber in der Sportpädagogik nach wie vor Auffassungen und Intentionen en vogue, „durch Sport“ zu irgendwelchen angenommenen Positivitäten zu erziehen. Dagegen steht das Prinzip einer nichtaffirmativen Erziehung der kritisch-konstruktiven Pädagogik (Gissel, 2019) ${ }^{13}$ sowie der Gedanke einer eigenständigen sinn- und wertvollen Körper-Kultur.

Für eine wissenschaftssystematische Verortung der Körperkultur müsse daher, so Werner, zwischen der empirischen Arbeit der Einzelwissenschaften und dem einigenden Band, bestehend aus den kulturellen Figurationen des Körperlichen, unterschieden werden.

\footnotetext{
13 Es lässt sich mit Adorno (1972, S. 107) darin begründen, dass niemand das Recht hat "von außen her Menschen zu formen" und "darüber zu entscheiden, wozu andere erzogen werden".
} 
Diese logische Scheidung kommt erst durch die Unterordnung der Ergebnisse der Einzelwissenschaften unter den Begriff Körperkultur zustande, ein Begriff, der selbständig ist, seinen Wert und seinen Inhalt nicht aus den Einzelwissenschaften bekommt.

Damit vertrat Werner eine Position, die in ganz ähnlicher Form 50 Jahre später von Grupe (1971, S. 15) in der Erstausgabe der Zeitschrift Sportwissenschaft vertreten wurde: Sportwissenschaft (Singular) sei als interdisziplinäre Wissenschaft $\mathrm{zu}$ konzipieren, der ihre „Problemstellungen von ihrem Objekt aus vorgegeben“ werden. Multidisziplinäre Sportwissenschaften (Plural) würden in eine „Addierung“ (S. 17) führen, die das Fach in „einzelwissenschaftlich-isolierte Problemstellungen“ (S. 15) zurückwerfe. Eine interdisziplinäre Sportwissenschaft müsse „die Sicht auf den Sport als einen Forschungsbereich, der unter Aspekten verschiedener Wissenschaften untersucht wird" (S. 16), lenken. Auch Kurz (1992) vertrat in seinem Disput mit Scherler (1992) eine solche Position: Eine Sportwissenschaft (Singular) benötige ein einigendes Prisma, einen allgemein anerkannten gemeinsamen Gegenstand, ansonsten zerfalle das Fach (Sportwissenschaften/Plural) in ein stückwerkartiges Konglomerat aus mehr oder weniger beliebigen Einzeldisziplinen. Für Kurz liegt dieses einigende Band in der pädagogischen Verantwortung, bei Werner im Kulturgedanken, der aber auch pädagogisch begriffen wird.

Mit seiner kulturtheoretischen Perspektive zeigt Werner zugleich eine Perspektive für das schwierige Normen- und Werteproblem der Sportpädagogik auf. Als Bereichspädagogik vermittelt sie ähnlich wie Kunst, Musik oder Literatur - „ästhetische oder wertorientierte Unterrichtsinhalte" (Prohl, 2013, S. 6). Dies führt, wie Prohl (2013, S. 11) zeigt, in ein Dilemma: Wenn sich die Sportpädagogik „am kritisch-rationalistischen Wissenschaftsverständnis der anderen sportwissenschaftlichen Teildisziplinen orientiert und empirische Forschung betreibt, ohne auf ihre normativen Grundlagen zu reflektieren, dann verliert sie ihren charakteristischen Gegenstandsbereich ,Er- ziehung und Bildung' aus den Augen, der stets auch normative Komponenten beinhaltet“. Orientiere sie sich nicht daran, so verkomme sie entweder zur „vorwissenschaftlichen Praxeologie der Leibeserziehung“ oder verlöre sich in „Empirielosigkeit und Praxisferne“; oder, wie Thiele (2013, S. 43) es ausdrückt, in „pädagogischer Folklore“.

Kultur habe immer etwas mit Werten zu tun, so Werner, und es sei Aufgabe einer Wissenschaft der Körperkultur, diese fachspezifischen Werte herauszuarbeiten, zu verbreiten und gegen konträre Wertvorstellungen zu verteidigen. Ein Institut für Körperkultur müsse „Werte schaffend und zerstörend sein; es kann sich nicht darauf beschränken, zu erklären, zu sichten und zu sammeln.“

Hier tritt als Moment, durch das für den Studierenden jenes Stückwerk der Einzelwissenschaften Sinn erhält, das zweite Ziel des Instituts für Körperkultur in Tätigkeit, der Wille zur Kulturgestaltung, der wie ein Brennpunkt alles Einzelwissen in sich sammelt und es wieder ausstrahlt, aber verändert, verarbeitet in ganz neuer Form. Das Einzelwissen, die Technik der Übungen verlieren hier ihren unmittelbaren Wert und damit auch das Dilettantische.

Aufgabe der Wissenschaft (von der Körperkultur) sollte es also sein, die Ergebnisse der Einzel-/Mutterwissenschaften zu sichten, zu ordnen und zu verarbeiten, um sie damit für die pädagogische Praxis der Körperkultur nutzbar zu machen ${ }^{14}$; es war somit eine Aufgabe des Auswertens - also eine normative Aufgabe nach den Kriterien und Maßstäben des Kulturbegriffes. Werner fuhr fort, dass die Auseinandersetzung mit Werten zwar keine Wissenschaft im strengsten Sinne sei, würde man einen solch strengen Maßstab jedoch an andere Disziplinen legen, so bliebe außer Mathematik kaum ein

\footnotetext{
14 Ganz ähnlich formuliert es Grupe (1971, S. 17):Ziel der Sportwissenschaft sei es, das Fach „im Sinne einer integrierenden Wissenschaft" zu einer Disziplin zu entwickeln, ,die die relevanten Ergebnisse anderer wissenschaftlichen Disziplinen verarbeitet, zusammenfaßt und unter ihrer spezifischen Fragestellung zu einem zusammenhängenden - wenngleich wohl nicht widerspruchsfreien-Ganzen integriert".
}

Fach an den Universitäten übrig. Somit habe auch ein kulturalistisch ausgelegtes Institut für Körperkultur seine Berechtigung im Fächerkanon von Universitäten verankert zu sein.

Die unglaubliche Modernität der Ausführungen Werners liegt weiterhin auch darin, dass er damit ein wesentliches Ergebnis des Positivismusstreites der 1960er Jahre vorweg gesehen hat. Wissenschaft kann nicht objektiv und wertfrei sein, jedes Fach muss vielmehr seine grundlegend wertbeladenen Erkenntnisvoraussetzungen und -interessen stets neu reflektieren.

Zentrale Aufgabe des Institutes sollte es nach Auffassung Walter Werners also sein, den eigenständigen Kulturcharakter der Leibesübungen zu erforschen und zu beschreiben. Er sah in Turnen, Sport, Gymnastik und Tanz unaustauschbare kulturelle Figurationen, vergleichbar mit Malerei, Musik und Dichtung, die ebenso ihren ureigenen Wert haben und nicht in den Dienst anderer angenommener Positivitäten gestellt werden dürfen. Werner war sich im Klaren darüber, dass er mit dieser Auffassung Konflikte heraufbeschwor. Er wies in seiner Denkschrift darauf hin, dass die Dozenten des Institutes eine besonders schwere Aufgabe hätten: Sie betreten völliges Neuland und müssten gegen Kreise ankämpfen, „die die Förderung der Leibesübungen auf ihre Fahnen geschrieben haben, aber dabei nur an das Äusserliche der Leibesübungen denken“.

Er selber bestand diesen Konflikt nicht. Er sah sich fortwährenden Anfeindungen des Sportmediziners Huntemüller ausgesetzt und wurde 1928 durch ein sorgfältig eingefädeltes Intrigenspiel, das sich aus den Akten gut rekonstruieren lässt (Gissel, 1995), aus seinem Amt gedrängt. Er wurde durch den erst 24-jährigen Hans Möckelmann ersetzt, der das Gießener Institut in die Zeit des „Dritten Reiches“ führte. Walter Werner verließ Gießen und baute sich eine Existenz als Landwirt und Atemtherapeut auf, in der er seine reformpädagogischen Vorstellungen verwirklichen konnte. 


\section{Kritisch-konstruktive Sportpädagogik - ein Ausblick}

Auf ihrem Weg zu einer „normalen Wissenschaft" hat die Sportpädagogik sich zunehmend an das Sanktions- und Gratifizierungssystem der nach ökonomischen Logiken funktionierenden Universitätslandschaft angepasst und versucht, sich durch drittmittelgestützte Effektforschung zu positionieren. Doch genau dabei verliert sie ihren ureigenen Gegenstand aus dem Auge. Als empirische Lehr-Lernforschung ist sie von sportpsychologischer oder trainingswissenschaftlicher Wirkungsforschung nicht mehr zu unterscheiden ${ }^{15}$. Als „vergessliche" Wissenschaft hat sie zugleich bildungstheoretische und anthropologische Fundierungen, wie sie insbesondere in der „Theorie der Leibeserziehung" der 1960er und 1970er Jahre erarbeitet wurden, entsorgt. Der heutige sportpädagogische (und fachdidaktische) Mainstream speist sich aus den angenommenen Motivlagen (,Sinnperspektiven“) des Sports. Die aktuellen Schulsportkonzepte sind „ohne pädagogische Umwege" aus der Scheinlogik des Faches entwickelt worden und sie verzichten auf „allgemeindidaktische und -pädagogische Bezüge", so Elflein (2007, S. 15). Entsprechend attestiert er ihnen „Konzepthaftigkeit“ ohne bildungstheoretische Fundierung.

Ihre Schwächen liegen a. m. S. vor allem darin, dass Sportpädagogik in erster Linie als ,eine Teildisziplin der Sportwis-

15 Cachay und Bähr (1992, S. 287) bemerkten schon vor 30 Jahren, dass die Sportpädagogik "eine ihr angemessene spezifische Wissenschaftstheorie" entwickeln und diese "selbstbewusst nach außen hin" vertreten müsse. Diese Diskussion über die Bestimmung der Identität habe "streng theoriegeleitet" vonstattengehen zu gehen (ebd., S. 283). Gleichwohl erscheint die von Cachay und Bähr favorisierte Theorie des "systemtheoretischen Konstruktivismus" für eine ethisch fundierte Pädagogik denkbarungeeignet. Für Systemtheoretiker sind menschlicher Geist und Seele nur ein funktionales biologisches System, das sich selbst organisiert und Umweltreize verarbeitet. Liebe, Hass, Leidenschaft, Wünsche etc., alles, was Menschlichkeit ausmacht, sind danach funktionslose Epiphänomene oder wie im Behaviourismus nicht operationalisierbares „verdecktes Verhalten“. senschaften“ (sic./Plural!) (Prohl, 2013, S. 6) verstanden und in ihrem fachspezifischen "Gewordensein“ (ebd. S. 7) erklärt wird. Dabei wird übersehen, dass die von Prohl (2013) identifizierten Probleme der Disziplin nicht nur für die Sportpädagogik, auch nicht nur für die ästhetisch - „wertorientierten“ Fächer (Musik, Kunst, Religion), sondern sich grundlegend für jede Pädagogik ${ }^{16}$ stellen. Bei dem verengten Blick nur auf das eigene Fach wird „die Grundstruktur pädagogischen Denkens und Handelns" (Benner, 2012, S. 8) übersehen. Von der Wissenschaftstheorie über empirische und geisteswissenschaftliche Forschung weiter zu didaktisch-methodischer Konzeptentwicklung bis hin zur pädagogischen Handlungspraxis gilt diese "Grundstruktur", die aufzeigt, worin sich alle ${ }^{17}$ „pädagogischen Sachverhalte und Problemstellungen von anderen unterscheiden und abgrenzen lassen “18 (Benner, 2012, S. 7). Die Sportpädagogik und ihre Fachdidaktik arbeiten sich seit Jahren an Problemen ab, die in der allgemeinen Pädagogik längst bildungstheoretisch fundiert gelöst wurden!

Bereichspädagogiken und Fachdidaktiken stehen in einer „nicht auflösbaren Beziehung zur Bildungstheorie“, so Klafki (1975, S. 90). Versuchen sie, sich selbstbezüglich aus ihrer Fachlogik zu entwickeln, entsteht ein „Torso“, „da sie in solcher Isolierung ihr eigentliches, nämlich ihr pädagogisches Thema gar nicht zu Gesicht“ bekommen (ebd.). „Fachdidaktische Entscheidungen können (...) nicht aus den einzelnen Fachwissenschaften abgeleitet werden" (Klafki, 2007, S. 88). Aufgabe der Fachdidaktik Sport ist es vielmehr, zu klären und zu zeigen, was die Sportvermittlung (insbesondere im

\footnotetext{
16 Wenig nachvollziehbar ist, warum Prohl $(2013$, S. 6) von "Erziehungswissenschaften (früher Pädagogik)" spricht und damit die Grundstruktur pädagogischer Praxeologie in Frage stellt.

17 Also auch in Kontext Sport.

18 Vorschläge, wie etwa von Brezinka (1978), die einheitliche Grundstruktur pädagogischen Denkens und Handelns in eine Philosophie der Pädagogik, eine (empirische) Wissenschaft der Pädagogik und in eine Praxis der Pädagogik aufzuteilen, sind von der Scientific Community entschieden zurückgewiesen worden.
}

Schulsport) „für gelingendes Menschsein in unserer Zeit" (Schmidt-Millard, 2006, S. 356) leisten kann und die Kategorie, an der dieses zu bemessen ist, heißt Bildung. Ein Schulsport, der den Anspruch erhebt, pädagogisch zu sein, muss sich also dem allgemeinen Bildungsauftrag der Schule ein- bzw. unterordnen. Der "Wert" des Schulsports ist ,entlang der Idee schulischer Bildung zu bestimmen" (Grupe, 2000, S. 17). Es gilt also ein Primat der allgemeinen Pädagogik! Daran hat sich eine wissenschaftstheoretische Konzeption der Sportpädagogik $\mathrm{zu}$ orientieren; sie kann nicht aus dem verengten Blick der Scheinlogik „der jüngere(n) problemgeschichtliche(n) Entwicklung der Sportpädagogik als Teildisziplin der Sportwissenschaften " entwickelt werden ${ }^{19}$ (Prohl, 2013, S. 7).

Zugleich darf sie aber auch die Dignität ihres Gegenstandes Sport (i. w. S.) nicht aus dem Auge verlieren. Es ist nicht irgendein Gegenstand! Sport ist mehr als nur körperliche Aktivität. Sport hat in seiner Performanz einen jeweils spezifischen phänomenalen Gehalt, jede Sportart hat ihre besondere Ästhetik, damit eine Bedeutung und einen Sinn. Und deshalb ist, wie Walter Werner schon vor 100 Jahren deutlich machte, Sport eine Kulturform, die zu bewahren und weiter zu entwickeln ist; die Kernaufgabe der Sportpädagogik besteht darin, diese Kultur mit ihren ästhetischen Gehalten den Heranwachsenden zu zeigen.

\section{Korrespondenzadresse}

\section{Norbert Gissel}

Fakultät Für Sportwissenschaft, Ruhr

Universität Bochum

Bochum, Deutschland

norbert.gissel@ruhr-uni-bochum.de

Funding. Open Access funding provided by Projekt DEAL.
19 Wenn man aus der Geschichte, oder dem was man dafür hält, quasi gesetzesmäßige Folgeentwicklungen („,aktuelle Entwicklungen und Problemlagen der Sportpädagogik", ebd.) ableitet, könnte man auch das als "Fehlschluss" (und zwarhistorischen) bezeichnen. 


\section{Einhaltung ethischer Richtlinien}

Interessenkonflikt. N. Gissel gibt an, dass kein Interessenkonflikt besteht.

Für diesen Beitrag wurden von den Autoren keine Studien an Menschen oder Tieren durchgeführt. Für die aufgeführten Studien gelten die jeweils dort angegebenen ethischen Richtlinien.

Open Access. Dieser Artikel wird unter der Creative Commons Namensnennung 4.0 International Lizenz veröffentlicht, welche die Nutzung, Vervielfältigung, Bearbeitung, Verbreitung und Wiedergabe in jeglichem Medium und Format erlaubt, sofern Sie den/die ursprünglichen Autor(en) und die Quelle ordnungsgemäß nennen, einen Link zur Creative Commons Lizenz beifügen und angeben, ob Änderungen vorgenommen wurden.

Die in diesem Artikel enthaltenen Bilder und sonstiges Drittmaterial unterliegen ebenfalls der genannten Creative Commons Lizenz, sofern sich aus der Abbildungslegende nichts anderes ergibt. Sofern das betreffende Material nicht unter der genannten Creative Commons Lizenz steht und die betreffende Handlung nicht nach gesetzlichen Vorschriften erlaubt ist, ist für die oben aufgeführten Weiterverwendungen des Materials die Einwilligung des jeweiligen Rechteinhabers einzuholen.

Weitere Details zur Lizenz entnehmen Sie bitte der Lizenzinformation auf http://creativecommons.org/ licenses/by/4.0/deed.de.

\section{Literatur}

Adorno, T. (1972). Erziehung zur Mündigkeit. Frankfurt a.M.:Suhrkamp.

Becker, F. (2019). Den Sport gestalten. Carl Diems Leben. 3. Aufl. (S. 317-341). Duisburg: Universitätsverlag, S. 317-341.

Beckers, E. (1987).Durch RückkehrzurZukunft? Anmerkungen zur Entwicklung der Sportpädagogik. Sportwissenschaft, 17(3), 241-257.

Benner, D. (2012). Allgemeine Pädagogik. Eine systematische-problemgeschichtliche Einführung in die Grundstruktur pädagogischen Denkens und Handelns. Weinheim, München:Beltz.

Bernett, H. (1965). Grundformen der Leibeserziehung. Schorndorf: Hofmann.

Brezinka, W. (1978). Metatheorie der Erziehung. München, Basel: Ernst Reinhardt.

Cachay, K., \& Bähr, H. (1992). Sportpädagogik wissenschaftliche Teildisziplin oder integrativer Kern der Sportwissenschaft? Sportwissenschaft, $22,283-302$.

Court, J. (2011). Warum scheiterte 1920 August Biers Antrag auf einen "Doktor der Leibesübungen?" Überlegungen zur Gründungsgeschichte der Deutschen Hochschule für Leibesübungen. Sportwissenschaft, 41, 91-99.

Court, J. (2014). Deutsche Sportwissenschaft in der Weimarer Republik und im Nationalsozialismus. Bd.2. Münster:LIT.

Dinckal, N. (2009). Der Körper als Argument - Die Deutsche Hochschule für Leibesübungen und die Produktion wissenschaftlicher Gewissheiten über den Nutzen des Sports. In M. Krüger (Hrsg.), Der deutsche Sport auf dem Weg in die Moderne (S. 173-198). Münster:LIT.
Eisenberg, C. (1999). English Sports und Deutsche Bürger. Paderborn: Schöningh.

Elflein, P. (2007). Sportpädagogik und Sportdidaktik. Baltmannsweiler: Schneider Hohengehren.

Giese, M. (2016). Inklusive Sportpädagogik. Kritische Überlegungen zu einer anthropologischen Fundierung. Sportwissenschaft, 46(2), 102-109.

Gissel, N. (1995). Vom Burschenturnen zur Wissenschaft der Körperkultur. Giessen: Ferber.

Gissel, N. (2006). Walter Werner: Ein Institut für Körperkultur an der Universität Gießen (1921). In J. Court \& E. Meinberg (Hrsg.), Klassiker und Wegbereiter der Sportwissenschaft (S. 105-111). Stuttgart:Kohlhammer.

Gissel, N. (2019). Kritisch-konstruktive Sportpädagogik. Studien zur Bildungstheorie und Didaktik des Sports. Baltmannsweiler:Schneider.

Grupe, O. (1971). Einleitung in die "Sportwissenschaft". Sportwissenschaft, 1(1), 7-18.

Grupe, O. (2000). Sporterziehung und Schulsportkultur. Sportunterricht, 49(1), 14-19.

Güldenpfennig, S. (1998). Noch einmal: Instrumentalisierung in der Sportpädagogik? Sportpädagogik 22(1), 3-4.

Janssen, J. (2006). Robert Werner Schulte: Eignungsund Leistungsprüfung im Sport. In J. Court \& E. Meinberg (Hrsg.), Klassiker und Wegbereiter der Sportwissenschaft (S. 135-142). Stuttgart: Kohlhammer.

Klafki, W. (1975). Studien zur Bildungstheorie und Didaktik. Weinheim:Beltz.

Klafki, W. (2007). Neue Studien zur Bildungstheorie und Didaktik. Weinheim: Beltz.

Kurz, D. (1992). Sportpädagogik als Teildisziplin oder integrativer Kern der Sportwissenschaft. Sportwissenschaft, 22, 145-154.

Kurz, D. (2011). Zum Verständnis des mehrperspektivischen Sportunterrichts. Fragen an Dietrich Kurz. In P. Neumann \& E. Balz (Hrsg.), Mehrperspektivischer Sportunterricht (Bd. 2, S. 16-24). Schorndorf: Hofmann.

Luh, A. (2018). Sportgeschichte 2020? Sportwissenschaftliche Positionierung und geschichtswissenschaftlicher Paradigmenwechsel. In A. Luh \& N. Gissel (Hrsg.), Neue Forschungen zur Kulturgeschichte des Sports (S. 9-36). Hamburg: Feldhaus.

Meinberg, E. (1987). Warum Theorien sportlichen Handelns Anthropologie benötigen! Sportwissenschaft, 17(1), 20-36.

Moraw, P. (1991). 1920 und 1990 - Zwei Jahre Gießener Universitätsgeschichte. Gießener Universitätsblätter.

Prohl, R. (2013). Sportpädagogik als Wissenschaftsdisziplin - Eine Standortbestimmung mit empirischen Ausblick.ZSF, 1(1), 5-30.

Scherler, K. (1992). Sportpädagogik - eine Disziplin der Sportwissenschaft. Sportwissenschaft, 22, 155-166.

Schmidt-Millard, T. (2006). Wolfgang Klafki:Studienzur Bildungstheorie und Didaktik (1963). In J. Court \& E. Meinberg (Hrsg.), Klassiker und Wegbereiter der Sportwissenschaft (S. 350-357). Stuttgart: Kohlhammer.

Thiele, J. (2013). Normale Wissenschaft - Die Sportpädagogik im Prozess der Normalisierung? In R. Messmer \& A. Gogoll (Hrsg.), Sportpädagogik zwischen Stillstand und Beliebigkeit (S. 27-46). Basel, Magglingen: Eigenverlag.

Volkamer, M. (2003). Sportpädagogisches Kaleidoskop. Hamburg:Czwalina. 